\title{
An Analysis of English Language Courses for Sino-UK University Engineering Programs
}

\author{
Peilin Kong ${ }^{1,2}$ \\ ${ }^{1}$ School of Humanity, Soochow University, Suzhou, China \\ ${ }^{2}$ International Education School, Suzhou University of Science \& Technology, Suzhou, China
}

Email address:

peilin04@163.com

To cite this article:

Peilin Kong. An Analysis of English Language Courses for Sino-UK University Engineering Programs. Communication and Linguistics Studies. Vol. 4, No. 2, 2018, pp. 45-53. doi: 10.11648/j.cls.20180402.14

Received: June 9, 2018; Accepted: June 26, 2018; Published: July 21, 2018

\begin{abstract}
This paper takes an example of English language teaching for Sino-UK university students who are the students at International Education School in Suzhou University of Science and Technology. Based on at least two-year English-related courses' teaching, the team leader and the foreign English teachers express their feelings and difficulties in this paper, the challenges English courses teachers face and how to overcome inherent shyness of Chinese university students, and conclude the practical and effective solutions to the problems respectively for these students in Sino-foreign programs.
\end{abstract}

Keywords: English Classes, Sino-UK Engineering Programs, Effective, Inherent Shyness, Challenges

\section{Introduction}

Suzhou University of Science and Technology (SUST) is an engineering-centered multi-disciplinary institution of higher education, covering such fields as engineering, science, liberal arts, and management. Centering upon undergraduate programs, concurrently adult continuation education, the University takes an active part in developing postgraduate programs and undertakes the responsibility of fostering practical and creative talents. SUST established International Education School (IES) in 2011, and offers 4 Joint-degree programs with University of South Wales (USW) in Construction Management, Mechanical Engineering, Logistics Management and Civil Engineering. Besides compulsory courses on respective disciplines offered by others correspondent schools, there is an English teaching team who is responsible for six more English language courses offered for the IES students, which are Academic Reading (I, II), Academic Writing (I, II), English Basic Skills (I, II), Speech \& Presentation, College English, IETLS. This English teaching team is composed one Chinese English teacher as a team leader and several English native teachers. Among these English language courses, College English and IELTS are conducted by Chinese teachers, and the rest four courses are implemented by English native teachers. Chinese teachers perform the test-related courses, College English for Chinese College English Test 4 \& 6, IELTS courses for the minimum IELTS 6.0 which meets the basic language requirement for USW international students. These five following parts show the challenges and solutions when teaching for IES students.

\section{Shyness}

Whilst the influence of culture is a reason for 'shyness' in the classroom, it by no means clears the smoke from this affair. Chinese students both male and female display a fondness for driving their intellectual faculties, for excelling in academic pursuits and demonstrating progress in their individual development. To stereotypically denounce them as 'shy' would be to ignore the multifaceted nature of the Chinese educational system and the influence of ancient cultural traditions that encourage academic excellence [1]. Humans as social creatures would certainly be unable to uplift themselves without overcoming natural 'shyness'. Thus, it is warming to see Suzhou students display vigorous energy when discussing abstractions with peers, easy-going communication when attempting to solve problems and displaying social maturity that in many ways rises above their Western peers. Shyness is just a stereotype, much like other stereotypes of Chinese students such as being unable to think critically or express 
free-thought.

However shyness is an important issue. Stereotypes are not demonstrative of reality yet they sometimes hint at a deep-rooted truth. This is because shyness can be a hindrance to student-teacher dialectics, a style of teaching deviant from the teacher-focused classroom. Chinese students most often follow the dictates of their teacher and pressure should occasionally be reversed to them so to force them to think about the nature of their learning. I argue that 'shyness' is the observation of them made from the Western philosophical tradition.

It may be that as Chinese students are conditioned to be more obedient to their teachers, it then creates an educational environment where critical inquiry is deemed unnecessary. In situations where the students should be expected to be bold and inquisitive they are not. Yet, in other situations they would display the enthusiasm and exuberance that young adults be expected. Therefore the issue is not of whether they are capable or incapable of answering questions when asked, but rather if it is in their interests to do so. Suzhou students and by extension Chinese students are just as intellectually inquisitive as their Western counterparts. The argue that the majority of them choose not to display this overtly.

As this study is limited to only the singular observations in the various schools of Suzhou city, it should not be taken as a representation of China and the rest of East Asia. Rather it is a microcosm of how the influence of culture manifests in a school classroom and how students are able to cope with the stresses it brings. The power of Education is indeed as such and so it is immensely intriguing to probe further.

\subsection{On the Nature of 'Shyness'}

'Shyness' must be fist defined in the Suzhou classroom. A Foreign teacher asking a question to the classroom may find the students are reluctant to answer. This typically occurs when the question is openly given to all of the students. It is especially likely to occur at the start of the lesson when the students are not 'warmed up'. Likewise in small groups one may find that several students are also hesitant to voice their opinions on matters, other than a few confident minorities. This can even occur after months of building relationships with students, where the teacher may know students on a first-name basis. This is the problem that 'shyness' will be identified.

For the purposes of this report, a model scenario is used to demonstrate 'shyness' in effect. This is called 'Open Group Question' scenario and shyness is one of the results that can occur in it. That is, for the teacher to meet a sea of blank eyes who do not answer. If one were to repeat and ask again then there still may not be any volunteers. Asking several times afterwards, selecting a specific student, displaying annoyance, exasperation or even wrath may later incur an answer. The students who may break the silence are usually those who are confident in their English, not the students most in need of learning. This demonstrates that some students may answer after much effort is exerted to encourage them to do so. This is the infamous 'shyness' of Chinese students in effect.
There are various approaches to English teaching all with their different advantages. Every Foreign teacher will know that the avenues one can pursue for excellent teaching are plentiful. Teaching of course, is an adaptive profession that thrives on innovation. For the purposes, I will utilize the own-branded Three-Phase Informational Schema which I find effective in the University classroom. This technique is based on the Socratic Method and relies upon student-teacher interaction in a large classroom setting. Its primary objective is for gauging the prevalence of shyness at the start of a lesson and also throughout if one can adapt it. This is useful as the Schema requires students to speak often. The secondary objective is to ensure gaining analytical information from students is simple, so the teacher can later tailor their teaching specifically for the class. For now the explicit shyness will be focused on the demonstrated in the Open Group Question scenario with the use of the Schema.

In conjunction to this, there are other situations where shyness is also prevalent in the classroom. This report argues that the 'Open Group Question' scenario is most useful for the study of shyness. The reason for this is because it offers the largest sample size of students. In smaller groups and even on a one-to-one basis it is common for shyness to be seen but I argue that due to the scale, the frequency of shyness occurring is more likely in an Open Group Question scenario. From this a satisfactory presentation of shyness in the classroom can be discerned and use this to make predictions for smaller groups.

\subsection{Frame of 'Shyness'}

The first part of the Schema where 'shyness' is most present is the 'Enquiry Phase'. In this phase, the teacher's objective is to ask an open question that students can answer appropriately. This can be done before, during or after the teaching of content. The timing depends on the teacher but after doing so the teacher is able to gain information about the students. If the teacher is unable to make use of this information then it can make teaching ineffective. This is because the teacher remains unaware as to what degree content is being absorbed by the students or not. Conversely if the teacher can make use of his enquiry then it will allow for a productive lesson. Thus, the 'Enquiry Phase' demonstrates the importance of a two-way dialogue to the students. Students should become accustomed to being constantly tested on their knowledge. This confers them to be accountable for their learning and which in turn allows opportunity for later clarification. Without an appropriate enquiry to the student's learning, teaching is difficult and cumbersome. An effective enquiry will seek to avoid that.

The second part of the Schema is the 'Reaction Phase'. This phase begins as soon as a student answers the question and a discussion is formed. It is important a broad a range of students are selected so to accurately gauge the class's competence with the content. By doing so, one can recognize the pace students should be learning at. From this the teacher will note if it is necessary to omit or extend concepts from the Lesson Plan. Highly positive reactions suggest that the teacher can immediately challenge students with more difficult 
content. Less positive reactions means the teacher needs to slow the pace of teaching, as students may not be following the lesson coherently. Thus the teacher is able to make adjustments based on this feedback. Through gaining answers from students one can tailor one's teaching style to fit the student's needs. This ensures teaching is effective and rapport can be built. Indeed, the 'Reaction Phase' is incredibly useful to prepare students for an effective teaching session.

The final part is the 'Review Phase' which is the most productive period of teaching. After gaining responses the teacher is able to review what has been discussed. A topic can be concluded and the teacher can begin the teaching of a new topic. From listening to students in the Reaction Phase, the teacher will also be able to deliver a well-tailored explanation of the concept they are trying to teach. By this point, most students should be aware of what it is that they should learn. Through keeping all the students on the same 'wavelength' by constantly reviewing topics, the teacher can efficiently communicate to them. As a result teaching at the Review Phase becomes fluid and from it learning is able to take place far more effectively.

Although shyness is visible in all phases it is most prevalent during the Enquiry Phase. Here students may not give an answer that would lead to an effective Reaction Phase. Many may not answer at all or if they do then the answer may be insufficient for the purposes of the Review Phase. Thus, it is evident that shyness can become a massive stumbling block for effective English teaching. The results of this are multifold and tenuous. Every teacher has their own unique style of teaching and it would be rare for them not to find shyness in their classroom. As such the Foreign Teacher's common feedback is that it becomes arduous to teach simple concepts, or that their successful Lesson Plan objectives are not completely actualized. Shyness unresolved can indeed be debilitating to the functioning of a productive classroom.

\subsection{Why 'Shyness' Occurs}

It is evident that Suzhou students are less adapted to vocally displaying their thoughts or opinions. There are several factors contributing to shyness in the Suzhou classroom. Finding the nature of these factors begs a formal study. This can be better assessed from the expeditionary lense of a skilled anthropologist, sociologist or historian. Such a study could help further explain the relative timidity of most Chinese students in comparison to counterparts in Western countries. Nonetheless this report can only be within the scope of the Suzhou classrooms with the deductions made from the Schema that is used.

One interesting observation of shyness is its increase in prevalence as students become older. The older students are, the more reluctance they show to answering questions vocally. If one observes younger children first then the differences are quite noticeable. For instance those in nursery or primary school are highly inquisitive about their lessons. A classroom of young students is typically loud and can be difficult to control. The challenge in these classrooms is that how does one teach such an energetic force of human beings? When compared to older students, those at the younger ages of 5-11 can certainly be described as 'lively'. The positive of this is if an excellent teacher can successfully implement their Lesson Plan objectives, then the young students have the potential to be highly receptive. Questions can be answered much more freely within this age group, an attribute that is clearly not as visible in older groups.

All children at young ages are curious about the world that inhabit. The late psychologist Ernest Becker has described young children as 'natural philosophers' [2] and there is a great deal of truth in this statement. Children will readily ask questions not because of any specific cultural influence or individual trait, but rather it is in their innate nature to do so. This is because a child's mind is the most absorbent for knowledge at their developmental ages. Therefore it is normal for the child to develop a healthy psyche through regular questioning of their observations. Anyone who has ever spent significant time with a young child would be accustomed to the question 'why?'. Even in tribal groups young children have often demonstrated the capability to be highly social and inquisitive from a young age as a means of survival [3]. By asking questions and creating conversations from those questions, children can learn about the world and how to best navigate it. As such young children of all cultures and ethnicities are naturally equipped to learn through the methods of question and answer. It is in the interests to fully utilize this in the classroom.

Indeed, young children thrive off the Positive Reinforcement that they can gain from 'getting the answer right' or 'asking the right thing'. It is crucial in their development not just as children to teenagers to adults, but also as students. Children require teaching not just on Mathematics or English, but also on the importance of a critical thinking. By facilitating their natural inquisitiveness, critical thinking can be delivered by the teacher through English teaching. Certainly the positive developments that result from teaching young children is a commonly held reason for teaching to be regarded a 'rewarding profession'.

The observatory lenses can then be placed on Middle School students and by association High School or University students. It is evident by this age, Chinese students become less forthright in their questioning and as evidenced by the Schema less inclined to answer questions. A vast array of theories and supplementary anecdotes can be utilized to explain the true nature of this. Yet, it is beyond the scope of this report to explain fully. It would not be appropriate to attempt at scrutinizing an entire culture's traits based on observations from only a tiny segment of that culture.

However a popular theory is utilized to briefly explain why students become more reserved as they age. One common idea is the importance of 'face' in Chinese culture. Though highly documented by anthropologists this is not unique to Chinese society. Similar cultural features have been observed not just in Eastern societies but also in Africa and South Asia [4]. It can also be argued that a variant of 'face' exists in the supposedly, pluralistic Western society. It is simply that people may avoid stating certain matters in public so as to avoid 
judgement or stigmatization from peers. A similar mechanism exists in Chinese society but to a much greater extent. Due to the importance paid to orderliness of state Confucianism, peoples influenced by Chinese culture are generally more reluctant to voice opinions in large groups. This is so to avoid creating a situation where conflict within the group can occur. Such a situation would be detrimental to harmony and group cohesion which Eastern values emphasize over individualism or free-thought. Therefore, Chinese people demonstrate more agreeableness and as a consequence less questioning in order to maintain relations with groups they identify with. This can be the form of what naive Westerners interpret as a lack of critical inquiry in people influenced by Chinese culture.

However, to place this view upon the entire people of China and the Sino-sphere would not be a sensible notion. Certainly amongst a large group such as a classroom or a group where the members are not familiar or friendly with each other can inquiry be limited. Yet between friends whom have more casual relationships such social etiquette is deemed unnecessary, even irritating. There is no immediate need to 'give face' or rather, there is no worry about losing face between those one enjoys the company of. It is something that requires consideration between people whom one is unfamiliar with.

This shows that the older one becomes in Chinese society the more aware they become of 'face' relative to other people around them. In the classroom this equates to students taking larger precautions when they are needed to express themselves vocally. Indeed, as the student becomes self-conscious of their peer's opinions then they will respond to the teacher at a less than optimal standard. Likewise as elder respect is a prominent aspect of Chinese culture this further complicates matters. As students age they begin to build a complex system of respect with their younger peers. In a classroom setting this occurs on the micro-scale, so much so that students develop psychological coping strategies such as remaining silent in order to avoid losing face to their peers. This is because social status in the classroom can be jeopardized irrespective of how intelligent or 'true' one is. As a result, the less pronounced social bonds are between students, the more shy they are likely to be.

For an older student they must warrant serious care towards their words in class. The student needs to maintain face in class not because they lack critical thought but because their social status depends on it. At a young age such matters are not important but as the student becomes more mature, responsible and further integrated into the adult world then so they must focus on self-preservation in the social arena. Therefore, a larger importance is seen placed on social relationships. This is because the utility of social circles is paramount, where a hypothetical friend of a friend's uncle could one day assist in one's economic mobility. Relationships become a form of capital which contains value in all parts of life in China, including the school classroom. The students must take care to not waste this and for the most diligent it may mean remaining silent in the classroom. So then effects of this can then be felt in the classroom as shyness.
In the modern West typically disassociation from oneself such cultural mechanisms by persuading ourselves that they are peculiarities of traditionalism. Any presumption is derided that not somehow the individuals that should think students to be. That is what may not be as special as what to be thought. That the self-importance each Westerner places in themselves somehow overrides our need for collective survival through social groups. Indeed in a social setting, Westerners' are just as affected by the importance of maintaining the reputations. Despite this, it must be remembered that the Western academic tradition since the days of Socrates has been one of pursuing rationalism and objectivity. Western educational establishments encourage students to argue their views in the classroom so as to deduce truth about the universe. So as Western students are taught to thrive from this tradition as a means to educate themselves, so too they are also less affected by shyness. This is apparent as they go to university where in fact they have more to gain through pursuing original thought with debate and intrigue. Superficially, the system of current education is more arguably meritocratic.

In China such a tradition has not existed to the scale of that in the West. In a limited form what can be seen in the traditional Imperial Examinations which were set from the late Han Dynasty until the Qing. Yet these examinations only selected a small segment of society and did not entirely test the examinee's on technical matters. One can argue that a reason for the fall of the Mandate of Heaven was because of the failure of the bureaucrats to incorporate critical inquiry into Chinese scientific progress. This was the most educated segment of society that at one time fostered much of the world's learning and as a result provided many of the inventions that what still be used today. Yet it before China's century of humiliation it was unable to innovate so to compete evenly with the technology of foreign powers. Thus the Opium Wars has been appeared, Sino-Japanese Wars, the Boxer Uprising and so on. Although this is a matter of historical perspective, it is clear the lingering influence from this style of education still continue in the classroom today.

\subsection{Solutions}

There is plenty one can do to overcome shyness. Having an understanding of how and why shyness occurs, operating with a basic understanding of not just macro-Chinese culture but the local culture, maintaining firm confidence in one's teaching abilities and building personal rapport with each of one's students are all important factors. However, it is beyond the scope of this essay to provide detailed solutions for this phenomenon. For such a study larger resources need to be entertained.

As the shyness could be seen amongst the students, to respond with the strategies to lessen its prevalence. In the classroom, the positive effects of the attempts could be noticed at nullifying it. However such strategies may not be appropriate for codification especially if they have not been peer-reviewed. Therefore, by no means a way is found to completely eliminate shyness. To do so would require a complete transformation of the culture the students hail from. 
Similarly expecting students to adapt to the Western standards completely is a losing battle. One can certainly influence them to overcome shyness and be able to give their opinion on matters. Yet it is a difficult task for Chinese students to utterly mirror their Western counterparts. Traditional and textbook-bound teaching styles often result in too little in EFL classrrom. The lack of a strong interactive atmosphere also appeared to be reflected in the student teachers' teaching in this study [5].

It is fortunate that inhabiting as a part of China that is rich in local culture and economic prosperity. This gives the people a great speaking confidence that is quite unlike other parts of China. It is evident that the students are positive and better equipped for the classroom then they would be in a more impoverished province. Likewise they display a sincere pride in the culture of the $\mathrm{Wu}$ state that once ruled Jiangsu and Zhejiang. This allows for stronger bonds between students and greater self-assurance in them individually. A similar yet more pronounced effect be found in the people of Shanghai where the material advantages of the 'Chinese Dream' have had a Westernizing effect on the people [6]. Indeed as many Chinese people consider Shanghai to be the bastion of Westernization in China it is certainly plausible that this would have a spillover effect in Suzhou. All of this assists greatly in overcoming shyness in the classroom.

However like Shanghai, Suzhou also wields many Chinese from other provinces. As such it is common to have a classroom with not just local Suzhou people but also the children of migrant workers. Likewise, different classes will yield different students each with their own talents and unique backgrounds. One cannot simply generalize culture or economic well-being towards excellence in learning. Such a micro-cultural argument would not be useful for overcoming shyness. Suzhou people are still Chinese and the importance of 'face' still remains a large part of their cultural identity which cannot be removed totally.

That is why refined teaching technique and personal rapport is important. It may be true that L2-based classes can create a psychological distance between teachers and students, and prevent the establishment of good relationships or rapport with every student [7]. Without constant self-evaluation of one's teaching skills, what techniques work or what does not then there is no way to improve a student's learning. If techniques are created in mind with removing shyness then this will naturally foster an engaging learning environment. From this the teacher can begin to understand each individual student and tailor teaching to their needs so to allow for critical thought. Yet, this is no simple task. Such is the nature of teaching that so much of it depends on the teacher's input. Nonetheless by keeping these matters in mind, one can certainly win over their Chinese students. From shyness to boldness is merely but a step away.

\subsection{Part Conclusion of 'Shyness'}

Shy students are a part of teaching in China. The prevalence of shy students increase as they become older and it becomes more difficult to encourage them to answer questions. This has been a very brief look into a deep topic. Perhaps this report provoked more questions than it did answer, but it is evident that one cannot create a classroom that interested in Western dialectics overnight. There are numerous strategies and approaches one can take to help alleviate shyness, but it is not something that one can remove entirely. Indeed it is a part of the landscape in the educational environment of China. Like the green mountains and the long rivers, to attempt at removing it from sight would be a near-villainous task. Instead what must to do is to learn how to adapt to it and how to work with it, not against it. That is but one way to become an excellent teacher in this mysterious civilization-state.

\section{Oral English Courses}

Although five years of teaching English in Chinese universities, the experience has been mostly concerned with General English and IELTS English. This paper aims to give an insight into the experiences teaching a course on Speech and Presentation, outlining the challenges and observations of teaching a more specialist subject in this environment.

Public speaking is a vital skill for professionals in every field, to study it as a subject in English thus also improving students level of English is an excellent opportunity for students to develop two closely linked skills in tandem, both of which will be vital for the students' future careers in university and beyond. It was also surprising that students who were not in any of my classes would also appreciate greetings and small talks in English [8].

The students are third year Engineering majors, the majority of whom are aiming to further their studies abroad.

This part deals with the main challenges faced by educators and students in this course. The majority of these are also applicable to many ESL environments such as different social and educational cultures, student vs teacher expectations, classroom dynamics, and so on. However, the combination of ESP and ESL has produced some interesting and novel challenges, creating the opportunity for more creative solutions.

\subsection{Frame of 'Oral English Course'}

In the first part discussion of the principal challenges faced during the course, as well as those inimical to the experience teaching in Chinese universities previously, discussion will be the more unfamiliar hurdles unique to a speech and presentation course.

Secondly the explanation to the solutions that what have been employed to face these challenges drawing on the experience of previous positions, and how to adapt the teaching skills to a new challenge

In the final section the proposals to some solutions aimed to employ in future. As with any new experience, there have been mistakes and these can be seen as an opportunity to improve and be more creative with ideas. 


\subsection{Lack of Textbook}

When designing this course the lessons should be complied from a mixture of online resources and resources that had been used in other courses, a book is found related to public speaking that has been used as a reference for myself, but did not include it as a requirement for the students. This did not prove to be a good strategy for several reasons.

Firstly, this was an unfamiliar situation to the students, although they have obviously come into contact with western teaching before, this seemed to throw them off and especially the weaker students often seemed confused as to what was expected of them.

In contrast to the own experience as a university student, the majority of students lack the initiative to research the books that should be recommended to them, let alone find their own materials. This is experienced this in other university situations that have been taught in China, but the fact that this was a course related to a special purpose rather than a more general English course exacerbated the problem.

The handouts and text that be given to them often ended up not being kept by students. Again this relates back to study skills, in spite of the instructions many students did not bring notebooks to class and often scribbled their notes on loose pieces of paper. This is assumed that being third years, they would realize that taking notes and keeping them organized is essential to learning a subject, but apparently in this case they did not realize this.

It is also suspected that the lack of a textbook gave students the impression that the course was somehow less 'serious' than their other courses and combined with their lack of study skills, had a highly negative effect on the students' motivation.

This may also be due to the students majors being in the field of engineering, coming from a humanities background myself and having more experience teaching Business and humanities related subjects, such a good idea has been come out about how engineering is taught. It may be the case that engineering students are not required to work independently or conduct research without more specific instructions due to the more linear nature of the skills required to succeed in the subject.

\subsection{Solution of Textbooks}

In future with a specialist subject that it a core text would be a priority. As well as a reading list, assignment will be at least one core text for students in future to serve as a workbook and will also help them to keep their work organized in the book and get a better sense of progress through the course.

Although the teacher is not a big fan of relying on a book as the teacher feels that a variety of sources gives students a better perspective and encourages a more acute mind by using the key skill of comparing and evaluating different sources, with regards to these students and this subject, it was a mistake.

Although it is haven't decided on which book to use, the teacher will probably use either 'Advanced Public Speaking. Dynamics and Techniques by Ruth Livingstone or The Art of Public speaking by Stephen B Lucas. From the lessons that it is have been done based on those books, they have both been very comprehensive and useful books, although they would still need to be adapted to the level of the students.

\subsection{Solution of Homework}

This seems to have a similar set of causes to the previous problem. The homework has been given to students in this course was fairly minimal and largely based around projects rather than linear assignments or exercises.

Again this did not suit the way that students were used to studying and the freedom that the teacher was attempting to give to them often translated into apathy and lack of engagement rather than encouraging them to use initiative.

As well as aiming to encourage independent study, to be honest one of the reasons for this was because since the course was new for me and I had to rethink the structure several times due to certain strategies and approaches not working I was not always able to come up with homework for the class because the teacher was not always $100 \%$ sure of what order the lessons would be in and what the students would be doing over the coming weeks. This was something that will be easier to avoid next year now that the course has a more stable structure in the view.

With regards to the students' study habits, it is also found that although the classwork was often unsuccessful with students often unable to complete the exercises and tasks that they were given in class, the teacher had much more success with the assignments that were given to them as homework. Often students who were very quiet and not engaged in classwork would produce surprisingly good work when given a clear task to do out of the class.

The atmosphere in the classrooms is often not conducive to study, although this is by no means unique to Chinese students, it does seem particularly acute in the universities that I have worked in. Because Chinese education tends to be based on a very teacher centered approach, the style of teaching that the teacher has been trained in where the majority of classwork is done in small groups of students with the teacher monitoring and offering support and advice rather than being the center of the lesson is often unfamiliar to students and can be a difficult way for them to work.

\subsection{Solution of Oral English Course}

Obviously the most obvious solution is simply to make sure that students are given a fairly straightforward task every single week to review the points covered in the lesson. As well as giving them a more tangible way to review their work, this will also give them the habit of studying and thinking about the classes, as well as an opportunity to study English in a different context.

There is also an interesting fairly new pedagogical concept called 'Flipped Classroom' which involves having students homework being based mostly on the next weeks class, researching the topic in the next weeks lesson. This is based on the idea that the actual lessons can then be much more focused on collaborative work rather than dispensing information. 
Although the concept was developed for language teaching, the teacher feels that this approach would be incredibly useful in this context as well. As I mentioned, the students often seemed much more confident with their homework than their classwork, so if they were able to prepare beforehand, they would get much more out of the actual lessons as they would have more confidence in using the skills that the course covers.

This was borne out by the assignments that the teacher did have the students complete, they responded much better to being given tasks to complete and then have me give feedback on than when they had to complete tasks using information given to them in that lesson.

It also lends itself well to the subject of Speech and Presentation because giving them tasks to prepare means that groups could prepare presentations to deliver in the class on the subject.

\section{Discipline}

Although with a couple of rare exceptions, the students are not unruly, trying to make them do work has been a constant struggle. Many of the students are addicted to mobile phone games and struggle to complete even simple tasks because of this.

\subsection{Problem Description}

In a class of $30-40$ students the teacher simply cannot keep an eye on everybody, and since they are adults it is inappropriate to shame them or shout at them. This also would be a bad idea both in that the class is based on giving presentations and that it is being taught in students second language, both of which scare people anyway without having an intimidating teacher.

Although the teacher has had several year- experience of teaching in Chinese universities and is very well aware of the challenge of large classes and the differences in educational culture, the teacher was surprised by the level of disengagement and lack of effort from many of these students.

As a teacher what is frustrating is that the third year classes are unbalanced against the good students and many of the more intelligent ones feel intimidated to even do any work in class due to the negative atmosphere in the classroom. Some of the brighter students have confided to me that they share the frustrations.

The teacher educators were seen as the authority; students tended to look for the best reply-turn to the type of explanations, requests, and contributions [9]. This problem has admittedly been compounded by the other issues that the teacher has previously mentioned in relation to the fact that the teacher has not been $100 \%$ happy with the course and since it is the first time to teach the subject, the structure of the curriculum is not always clear or obvious to students. However, the teacher has also experienced students simply 'shutting down' rather than engaging with the lesson when the teacher attempts to explain the task that the teacher was giving them in several increasingly simple ways so that they can at least make an attempt to do something in the lesson.

The experience teaching English for a long time and the own beliefs about education have led the teacher to believe that often when students are 'lazy' they are actually lacking in confidence or unsure of what they are supposed to be doing, rather than being obstinate. Although that is not to say that the blame lies entirely with the teacher, the problem with mobile phone addiction and just poor attitude are not something that the teacher can fix seeing them for two periods in a week, and not something that the teacher is qualified to deal with, but by the same token, the teacher can understand why students sometimes become disengaged with classes where the aims are not clear to them.

\subsection{Solution}

The main plan for the future is to make use of self-study and the 'flipped classroom' approach that have been mentioned before. The two causes for the discipline problems, lack of understanding the task, and general poor attitude can both be addressed by this.

With regards to not understanding the task, if students are given an introduction to the task and allowed to prepare for the lesson in their own time and using their own methods, they will feel more confident to complete the tasks during class.

With regards to the responsibilities of the student, if students come to class without having done the homework and unable to participate in the lesson, this makes it much easier for the teacher to keep track of which students are not performing their duty, and then act accordingly with regards to counselling said students, or failing that giving them a final participation grade reflecting their poor performance at the end of the semester.

\section{English Study Skills}

Again this was a problem that have been anticipated, but not to the extent that it hindered the classes. It is found that many of what has been considered basic study skills for university students to be sorely lacking in these classes.

\subsection{Lack of Language Study Skills}

The most surprising for me was the extent to which students struggled with active listening. As is mentioned before, a great deal of students come to class without a notebook or even a pen to take notes or do some written work if required. It has been explained to them that the teacher cannot produce handouts every week for large classes, but for some reason a great deal of them seem completely unable to grasp this. Handouts showed an increased level of understanding of the lectures through advance learning and active participation in discussion [10].

Rather than cooperating with one another during group exercises, they would tend to simply switch off when they weren't given a very specific task to do. Although this is not true for all of them, as groups they lacked even the most basic skills with regards to teamwork and often struggled to work 
together to complete even the simplest of tasks as a group.

One of the reasons for this that I have identified through the teaching this year has been the way that the students have organized themselves as a group. Every class has some very dominant 'leaders' who are active in class, and as what have been learned, also outside of class, and this has created a class dynamic which the less dominant ones have resigned themselves to becoming incredibly passive, to the point of inertia.

Additionally, when the teacher has given out tasks to students to work independently, many of them have not only not understood, but have pretended to understand to avoid having to do work.

There have also been a number of smaller problems such as a lack of understanding how to reference sources, organize a coherent piece of text, ask questions etc.

\subsection{Solutions}

It has already done this out of necessity, but the teacher will plan to do it more in future. The solution to this problem is simply that the students need to be taught these things. I have a class on active listening as part of the course, but the teacher did not teach it until a fair way into the lesson, on reflection the listening class should have come much earlier in the course since it is something that was one of the most relevant subjects that the teacher taught with regards to this course.

Likewise, what should be thought that some general classes on Study Skills will be incorporated into the class in future. The first lesson introducing the course includes an outline of the expectation of students' role in the class, but having experienced so many problems with students fundamental study skills, it is believed that the teacher should dedicate much more of the curriculum to this problem. Especially since many of the students are planning to study abroad next year and will experience problems with the study habits that could be seen from them.

\section{Syllabus Structure}

\subsection{Unfamiliar with Syllabus Structure}

This year it is divided the course over two semesters into Informative Speaking and Persuasive Speaking with one semester dedicated to each. The rationale was that since the art of Public Speaking is usually seperated into these two categories, it would make sense to do one semester on each. The teacher also included more general skills over the semesters, but overall it was seperated into semester one covering informative speaking, and semester two covering persuasive speaking. They are ordered like this because informative speaking is more familiar and accessible than persuasive speaking as a rule.

It hasn't been found this to be entirely successful, although they are separate to some degree, many of the skills are of course crucial to both of them. To some extent I found that this division was an artificial one and the teacher may have confused students by mankind this distinction.
Another problem with the way that should be structured the syllabus was that 'front loaded'. It is a bit too much with a lot more skills lessons in the first semester. One of the reasons for this as because the 'Persuasive speaking' half of the course had a lot more kinds of public speaking to focus on, whereas the Informative speaking half as has been mentioned was a much more familiar type of work for students

\subsection{Solutions}

It will be rearranged the syllabus so that the division is not so pronounced. The division is useful, but not as central to the subject as what had been thought when planning it in September.

In hindsight it is not so important that students are even aware of the difference to such a degree as to have such a pronounced divide. And in addition, this will allow me to mix up the lessons a bit more to reduce students becoming fatigued by studying similar topics for a long time.

\section{Conclusion}

From the two native English teachers and one Chinese English teachers' feedback to the IES students which are involved in the Sino-UK University Program, there are still a lot unsolved in English learning and skill enhancing. IELTS is the basic requirement for the students and oral communication and culture permeation are prime importance. For English teachers both domestic and abroad, this has been a very challenging class to teach, as a new subject for us, a number of decisions have been made which upon reflection could be improved on. The way that the work and information was presented to the students has not always been the most efficient or effective, and there are a number of areas of planning where the knowledge that much has been gained this year will improve the course a great deal.

The textbooks, homework and the way the syllabus is structured all have some problems which in hindsight could be executed more effectively.

As well as the challenges of teaching a new subject, some problems also appeared which will be familiar to anyone teaching in Chinese university such as lack of student motivation, and differences in the academic culture and approach between the approach to teaching and their method of learning.

Despite the many challenges, there has been much constructive work done, and some useful insights have been gained which will help us to improve the teaching in this subject and in general in the future. English and culture courses would also be more effective under the effort by the teachers, students and Chinese universities.

\section{Acknowledgements}

This paper is supported by Jiangsu Provincial Education Department, Grant Number is 2016SJB740031. And thank for Nicholas and Jackies' devotion for this paper. Both of them are native English language teachers for SUST and have given a lot of feedback and teaching experience to me. 


\section{References}

[1] Henderson, S. and Chan, A. (2005). Career Happiness Among Asian Americans: The Interplay Between Individualism and Interdependence. Journal of Multicultural Counseling and Development, 33(3), pp. 180-192.

[2] Becker, E. (1973). The denial of death. New York: Free Press.

[3] Diamond, J. (2012). The world until yesterday. New York: Viking.

[4] Mauss, M. and Evans-Pritchard, E. (1967). The gift. New York: Norton.

[5] Gan, Zhengdong; Yang, Chi Cheung Ruby. (2018). How Prepared are the Preservice ESL Teachers to Teach: Insights from University Supervisor Feedback. Journal of Asia TEFL. Volume: 15. Issue: 1. Pages: 99-117.

[6] Yi, Y. (2013). Youth urged to contribute to realization of

"Chinese dream" - Xinhua | English.news.cn. [online] News.xinhuanet.com. Available at: http://news.xinhuanet.com/english/china/2013-05/04/c 13235 9537.htm [Accessed 4 Jan. 2016].

[7] Sato, Rintaro. (2018). Examining EFL Teachers' Non-verbal Behaviors in English-medium Lessons.

[8] Journal of Asia TEFL Volume: 15 Issue: 1 Pages: 82-98.

[9] Balsemão Oss, Débora Izé (201806). The Relevance of Teachers' Practical Knowledge in the Development of Teacher Education Programs. Profile Issues in Teachers` Professional Development Volume: 20 Issue: 1 Pages: 167-178.

[10] Lucero, Edgar; Scalante-Morales, Jeesica. (201801). English Language Teacher Educator Interactional Styles: Heterogeneity and Homogeneity in the ELTE Classroom. How Volume: 25, Issue: 1, Pages: 11-31.

[11] Do, Suk-Jin. (2018). The Effect of flipped learning on ETP TOEIC class. Korean Journal of General Education, Volume: 12. Issue: 2 Pages: 149-167. 